\title{
PRICING OF QUANTO OPTION UNDER THE HULL AND WHITE STOCHASTIC VOLATILITY MODEL
}

\author{
Jiho Park, Youngrok Lee, And Jaesung Lee
}

\begin{abstract}
We use a power series expansion method to get an analytic approximation value for the quanto option price under the Hull and White stochastic volatility model, which turns out to be accurate enough by comparing with the simulation prices using Monte Carlo method.
\end{abstract}

\section{Introduction}

A quanto is a type of financial derivative whose pay-out currency differs from the natural denomination of its underlying financial variable. Its main purpose is to provide exposure to a foreign asset without taking the corresponding exchange rate risk. A quanto option is a foreign currency stock option whose payoff is converted into a domestic currency at maturity at a predetermined foreign exchange rate. The profit of quanto option is worked out a particular currency, but the pay-out of the quanto option is made by cash settlement of fixed exchange rate for another currency.

Stochastic volatility models are frequently used in pricing various kinds of European options. The most famous and popular stochastic volatility models include the Hull and White [6], the Stein and Stein [7], the Heston [5] ones. Their main purpose is to resolve a shortcoming that the Black-Scholes' constant volatility model which cannot explain long-observed features of the implied volatility surface such as volatility smiles and skews. For that reason, in valuing a quanto option it is natural to consider a stochastic volatility model.

Despite its importance, very little research has been done on pricing a quanto option using a stochastic volatility model primarily due to the sophisticated stochastic process for underlying assets and volatilities as well as the difficulty of finding analytic form of a quanto option price.

To mention some of the related previous work, Ball and Roma [4] examined alternative methods for pricing options when the underlying security volatility is stochastic.

Received July 19, 2012

2010 Mathematics Subject Classification. Primary 91B25, 91G60, 65C20.

Key words and phrases. quanto option, stochastic volatility model, Hull and White model, correlation expansion method. 
Alós [1] used Malliavin Calculus to construct option pricing approximation formulas under the Hull and White stochastic volatility model. Antonelli and Scarlatti [2] developed the methods of Alós to find a new approach for solving the pricing equations of European call options under stochastic volatility models by expressing the price in terms of a power series of the correlation parameter between the processes driving the dynamics of the price and of the volatility.

And then more recently, Antonelli, Ramponi and Scarlatti [3] adapted the methods of expanding and approximating the theoretical evaluation formula with respect to correlation parameters by Antonelli and Scarlatti [2] to find a new and analytic method valuating exchange options with random volatilities, which gave a strong motivation to our research.

This paper, we use the methods used in [1], [2] and [3] to find a series expansion formula to approximate the quanto option value with Hull and White stochastic volatility model. The methods used in [1] and [2] find series expansions of general option and exchange option with stochastic volatility models. We derived a series expansion pricing formula of quanto option using Hull and White stochastic volatility model with non-zero correlation. Then, we show that this is a good approximation by comparing our results with Monte Carlo simulation method.

We introduce some preliminary materials on Hull and White stochastic volatility model in Section 2. Then, in Section 3, we find an approximate value of the quanto option price by way of a PDE and a correlation expansion method. Theorem 3.3 is the main result of the paper. In Section 4, we show that simulation results using Monte Carlo method are close to our analytic approximation in both zero correlation and non-zero correlation cases.

\section{Preliminaries}

\subsection{Hull and White stochastic volatility model}

Assume that $S_{t}$ is a stock price, $\sqrt{u_{t}}$ is a volatility of the stock price and $B_{t}$ and $Z_{t}$ are standard Brownian motions. In risk-neutral world, using the Hull and White stochastic volatility model, we have

$$
d S_{t}=r S_{t} d t+\sqrt{u_{t}} S_{t} d B_{t}, \quad d u_{t}=\mu u_{t} d t+\xi u_{t} d Z_{t},
$$

where $r$ is a riskless interest rate and $\mu$ and $\xi$ are constants. Put $\sigma_{t}=\sqrt{u_{t}}$ and applying Itô formula gives

$$
\begin{aligned}
d \sigma_{t} & =\frac{\partial \sigma_{t}}{\partial u_{t}} d u_{t}+\frac{1}{2} \frac{\partial^{2} \sigma_{t}}{\partial u_{t}^{2}}\left(d u_{t}\right)^{2} \\
& =\frac{1}{2}\left(\mu-\frac{1}{4} \xi^{2}\right) \sigma_{t} d t+\frac{1}{2} \xi \sigma_{t} d Z_{t} .
\end{aligned}
$$

We may rewrite the equation (1) as

$$
d S_{t}=r S_{t} d t+\sigma_{t} S_{t} d B_{t}, \quad d \sigma_{t}=\tilde{\mu} \sigma_{t} d t+\tilde{\xi} \sigma_{t} d Z_{t} .
$$




\subsection{Quanto option with stochastic volatility}

Let $[0, T]$ be a time interval, and let $(\Omega, \mathcal{F}, \mathbf{P})$ be a complete probability space on which standard Brownian motions $W_{t}, Z_{t}, B_{t}$ and $\tilde{B}_{t}$ are defined. Applying the modified Hull and White stochastic volatility model (2) to stock and foreign exchange rate dynamics. Let $S_{t}$ be a stock price in foreign currency and $F_{t}$ be a foreign exchange rate which is an amount of domestic currency value per one foreign currency value. For some constants $\mu_{S}, \mu_{F}, \eta_{1}, \eta_{2}, \xi_{1}$, $\xi_{2}$, we can obtain the following equations.

$$
\begin{aligned}
d S_{t} & =\mu_{S} S_{t} d t+v_{t} S_{t} d W_{t}, & & d v_{t}=\eta_{1} v_{t} d t+\xi_{1} v_{t} d B_{t}, \\
d F_{t} & =\mu_{F} F_{t} d t+\sigma_{t} F_{t} d Z_{t}, & & d \sigma_{t}=\eta_{2} \sigma_{t} d t+\xi_{2} \sigma_{t} d \tilde{B}_{t} .
\end{aligned}
$$

Here, for some constant correlations $\rho, \nu$ and $\beta$, the followings hold

$$
\begin{gathered}
Z_{t}=\rho W_{t}+\sqrt{1-\rho^{2}} \bar{W}_{t}, \quad B_{t}=\nu W_{t}+\sqrt{1-\nu^{2}} \tilde{W}_{t}, \\
\tilde{B}_{t}=\beta \rho W_{t}+\beta \sqrt{1-\rho^{2}} \bar{W}_{t}+\sqrt{1-\beta^{2}} \hat{W}_{t},
\end{gathered}
$$

where $W_{t}, \bar{W}_{t}, \tilde{W}_{t}, \hat{W}_{t}$ are mutually independent standard Brownian motions. And the payoff of the quanto option at maturity time $T$ is

$$
\text { Payoff }=F_{0} \max \left[S_{T}-K, 0\right],
$$

where $F_{0}$ is a predetermined foreign exchange rate, $S_{T}$ is a foreign stock price at maturity $T$ and $K$ is a strike price in foreign currency.

\subsection{Qunato adjusted stock price dynamics}

In risk-neutral world, the foreign exchange dynamics for domestic currency with stochastic volatility $\sigma_{t}$ has a form as follows

$$
d F_{t}=\left(r_{d}-r_{f}\right) F_{t} d t+\sigma_{t} F_{t} d Z_{t},
$$

where, $r_{d}$ is a riskless domestic interest rate and $r_{f}$ is a riskless foreign interest rate. In real world, let $v_{t}$ be a volatility then by Itô lemma

$$
d\left(S_{t} F_{t}\right)=S_{t} F_{t}\left(\mu_{S}+\mu_{F}+\rho v_{t} \sigma_{t}\right) d t+S_{t} F_{t}\left(v_{t} d W_{t}+\sigma_{t} d Z_{t}\right) .
$$

From the above equation, in risk-neutral world for domestic currency

$$
d\left(S_{t} F_{t}\right)=r_{d} S_{t} F_{t} d t+S_{t} F_{t}\left(v_{t} d W_{t}+\sigma_{t} d Z_{t}\right) .
$$

On the other hand, in domestic currency

$$
d\left(\frac{1}{F_{t}}\right)=\left(r_{f}-r_{d}+\sigma_{t}^{2}\right) \frac{1}{F_{t}} d t-\frac{\sigma_{t}}{F_{t}} d Z_{t} .
$$

In risk-neutral world, using equations (6) and (7), we can find stock price dynamics in domestic currency as follows

$$
\begin{aligned}
d S_{t} & =d\left(S_{t} F_{t} \cdot \frac{1}{F_{t}}\right) \\
& =S_{t} F_{t} d\left(\frac{1}{F_{t}}\right)+\frac{1}{F_{t}} d\left(S_{t} F_{t}\right)+d\left(S_{t} F_{t}\right) d\left(\frac{1}{F_{t}}\right)
\end{aligned}
$$




$$
=\left(r_{f}-\rho v_{t} \sigma_{t}\right) S_{t} d t+v_{t} S_{t} d W_{t} .
$$

\subsection{Preliminary lemmas}

The well-known Duhamel's principle and the classical Feynman Kaĉ formula play important roles in the paper. Using the Feynman Kaĉ formula, we get the main PDE. After transforming inhomogeneous equations to the integral of homogeneous equations by Duhamel's principal, we can apply the Feynman Kaĉ formula again to the homogeneous equations to get the integral of expectation of random variables. Now, we introduce the Duhamel's principle and the Feynman Kaĉ formula.

Lemma 2.1 (Duhamel's principle). Consider a inhomogeneous equation for a function

$$
c:[0, T] \times \mathbf{R}^{n} \rightarrow \mathbf{R}
$$

with a terminal value problem

$$
\left\{\begin{aligned}
\frac{\partial}{\partial t} c(t, y)+L c(t, y) & =f(t, y) \\
c(T, y) & =0
\end{aligned}\right.
$$

where $L$ is a linear differential operator. The solution of this equation is

$$
c(t, y)=-\int_{t}^{T} p(t, y ; s) d s,
$$

where $p(t, y ; s)$ is a solution of the following homogeneous equation with terminal condition

$$
\left\{\begin{aligned}
\frac{\partial}{\partial t} p(t, y ; s)+L p(t, y ; s) & =0 \quad \text { for } t<s \\
p(s, y ; s) & =f(s, y) \quad \text { for } t=s .
\end{aligned}\right.
$$

Lemma 2.2 (Feynman Kaĉ formula I). Suppose that $f$ has a continuous derivative of order 2 and $q$ has a continuous derivative of order 1 on $\mathbf{R}^{n}$. Assume that $q$ is lower bounded. Put

$$
c(t, y)=E\left[e^{-\int_{t}^{T} q\left(Y_{s}\right) d s} f\left(Y_{T}\right)\right],
$$

where $Y_{t}$ is the n-dimensional Itô diffusion of the following form

$$
d Y_{t}=b\left(Y_{t}\right) d t+\sigma\left(Y_{t}\right) d B_{t}, \quad d B_{t}^{i} d B_{t}^{j}=\rho_{i j} d t .
$$

Then

$$
\begin{aligned}
\frac{\partial c}{\partial t}+A c & =q c ; \quad t>0, y \in \mathbf{R}^{n}, \\
c(T, y) & =f(y) ; \quad y \in \mathbf{R}^{n},
\end{aligned}
$$

where

$$
A c=\sum_{i} b_{i} \frac{\partial c}{\partial y_{i}}+\frac{1}{2} \sum_{i, j} \sigma_{i} \sigma_{j} \rho_{i j} \frac{\partial^{2} c}{\partial y_{i} \partial y_{j}}
$$


The following lemma is the converse version of Lemma 2.2.

Lemma 2.3 (Feynman Kaĉ formula II). Suppose that $f$ has a continuous derivative of order 2 and $q$ has a continuous derivative of order 1 on $\mathbf{R}^{n}$. Assume that $c(t, y)$ is bounded on $[0, T] \times \mathbf{R}^{n}, q$ is lower bounded and equations (10) and (11) hold then the solution can be written as an expectation

$$
c(t, y)=E\left[e^{-\int_{t}^{T} q\left(Y_{s}\right) d s} f\left(Y_{T}\right)\right],
$$

where $Y_{t}$ is the $n$-dimensional Itô diffusion of the form (9).

\section{Quanto option value by correlation expansion}

\subsection{PDE for option price}

Next we find a PDE for quanto option price $c(t, x, v, \sigma, \rho, \beta, \nu)$ in foreign currency with terminal condition. In general, when we value a quanto option price in domestic currency associated with the payoff equation (5), after calculating the option price in foreign currency, we multiply the predetermined foreign exchange rate $F_{0}$ to the foreign currency option price. For easy calculating, the option price $c(t, x, v, \sigma, \rho, \beta, \nu)$ is just calculated in foreign currency value. Or, we regard the predetermined foreign exchange rate as being fixed by 1 . Later, we will multiply the foreign exchange rate to the option price in foreign currency in order to obtain the option price in domestic currency that we want. We get the PDE by applying the Feynman Kaĉ formula directly.

Theorem 3.1. Let $[0, T]$ be a time interval. Under the model defined by (3) and (4), the European quanto call option price $c(t, x, v, \sigma, \rho, \beta, \nu)$ with maturity $T$ and strike price $K$ in foreign currency satisfies the following partial differential equation.

$$
\left\{\begin{array}{l}
\frac{\partial c}{\partial t}+\frac{1}{2}\left(v^{2} \frac{\partial^{2} c}{\partial x^{2}}+\xi_{1}^{2} v^{2} \frac{\partial^{2} c}{\partial v^{2}}+\xi_{2}^{2} \sigma^{2} \frac{\partial^{2} c}{\partial \sigma^{2}}\right) \\
+\nu \xi_{1} v^{2} \frac{\partial^{2} c}{\partial x \partial v}+\beta \rho \xi_{2} v \sigma \frac{\partial^{2} c}{\partial x \partial \sigma}+\nu \beta \rho \xi_{1} \xi_{2} v \sigma \frac{\partial^{2} c}{\partial v \partial \sigma} \\
+\left(r_{f}-\rho v \sigma-\frac{1}{2} v^{2}\right) \frac{\partial c}{\partial x}+\eta_{1} v \frac{\partial c}{\partial v}+\eta_{2} \sigma \frac{\partial c}{\partial \sigma}=r_{d} c \\
c(T, x, v, \sigma, \rho, \beta, \nu)=\max \left(e^{x}-K, 0\right)
\end{array}\right.
$$

Proof. We use the stock price dynamics (8) with the Hull and White stochastic volatility model and put $X_{t}=\ln S_{t}$, we can find following SDEs

$$
\begin{aligned}
d X_{t} & =\left(r_{f}-\rho v_{t} \sigma_{t}-\frac{1}{2} v_{t}^{2}\right) d t+v_{t} d W_{t} \\
d v_{t} & =\eta_{1} v_{t} d t+\xi_{1} v_{t} d B_{t} \\
d \sigma_{t} & =\eta_{2} \sigma_{t} d t+\xi_{2} \sigma_{t} d \tilde{B}_{t}
\end{aligned}
$$


The payoff of the option in foreign currency is

$$
f\left(S_{T}\right)=\max \left[S_{T}-K, 0\right]
$$

or

$$
f\left(e^{X_{T}}\right)=\max \left[e^{X_{T}}-K, 0\right], \quad K \in \mathbf{R} .
$$

In the absence of arbitrage opportunities, the option price $c(t, x, v, \sigma, \rho, \beta, \nu)$ at initial time $t$ is

$$
\begin{aligned}
c(t, x, v, \sigma, \rho, \beta, \nu) & =E\left[e^{-r_{d}(T-t)} f\left(e^{X_{T}}\right)\right] \\
& =E\left[e^{-\int_{t}^{T} r_{d} d u} f\left(e^{X_{T}}\right)\right] .
\end{aligned}
$$

Applying the Feynman Kaĉ formula (Lemma 2.2) to the above equation and using equations (13)-(15), we can find following equations directly

$$
\begin{gathered}
\frac{\partial c}{\partial t}+\left(r_{f}-\rho v \sigma-\frac{1}{2} v^{2}\right) \frac{\partial c}{\partial x}+\eta_{1} v \frac{\partial c}{\partial v}+\eta_{2} \sigma \frac{\partial c}{\partial \sigma} \\
+\frac{1}{2}\left(v^{2} \frac{\partial^{2} c}{\partial x^{2}}+\xi_{1}^{2} v^{2} \frac{\partial^{2} c}{\partial v^{2}}+\xi_{2}^{2} \sigma^{2} \frac{\partial^{2} c}{\partial \sigma^{2}}\right) \\
+\nu v^{2} \xi_{1} \frac{\partial^{2} c}{\partial x \partial v}+\beta \rho \xi_{2} v \sigma \frac{\partial^{2} c}{\partial x \partial \sigma}+\nu \beta \rho v \xi_{1} \xi_{2} \sigma \frac{\partial^{2} c}{\partial v \partial \sigma}=r_{d} c, \\
c(T, x, v, \sigma, \rho, \beta, \nu)=f\left(e^{x}\right)=\max \left[e^{x}-K, 0\right] .
\end{gathered}
$$

We want to calculate power series expansion formula of quanto option price function $c(t, x, v, \sigma, \rho, \beta, \nu)$. From PDE (12) we can find the PDEs which are derivatives for each correlations $\rho, \beta, \nu$.

\subsection{Taylor series expansion}

Let $[0, T]$ be a finite time interval. For $t, s \in[0, T], t$ is initial time and less than or equal to $s$. From (13), integral form of $X_{s}$ is

$$
X_{s}=x+r_{f}(s-t)-\int_{t}^{s}\left(\rho v_{u} \sigma_{u}+\frac{1}{2} v_{u}^{2}\right) d u+\int_{t}^{s} v_{u} d W_{u} .
$$

Let $\mathcal{F}_{s}$ be a filtration which is generated by the volatilities $v_{u}$ and $\sigma_{u}(t \leq u \leq s)$ then the distribution of $X_{s}$ conditionally on $\mathcal{F}_{s}$ is, for a normal distribution $N$,

$$
X_{s} \mid \mathcal{F}_{s} \sim N\left(x+r_{f}(s-t)-\int_{t}^{s} \rho v_{u} \sigma_{u} d u-\frac{1}{2}\langle M\rangle_{s},\langle M\rangle_{s}\right),
$$

where $M_{s}$ is denoted by the martingale $\int_{t}^{s} v_{u} d W_{u}$ and

$$
\langle M\rangle_{s}=\int_{t}^{s} v_{u}^{2} d u
$$

is its quadratic variation.

A simple modification of the Black-Scholes formula gives explicitly the value of a quanto call option. Since the underlying asset price $S_{t}$ is lognormally distributed conditionally on $\mathcal{F}_{t}$. Let $Q_{B S}$ be a Black-Scholes quanto option 
price formula in foreign currency. As we mentioned earlier, the option price that we calculate is not for domestic currency but for foreign currency. And the stock price dynamics for valuing option price is quanto adjusted. Therefore, for the strike price $K>0$, we have

$$
\begin{aligned}
Q_{B S}(t, x, v, \sigma) & =E\left[e^{-r_{d}(T-t)} \max \left(e^{X_{T}}-K, 0\right)\right] \\
& =e^{x+\left(r_{f}-r_{d}\right)(T-t)-\int_{t}^{T} \rho v_{u} \sigma_{u} d u} N\left(d_{1}\right)-K e^{-r_{d}(T-t)} N\left(d_{2}\right),
\end{aligned}
$$

where $N(\cdot)$ is the standard normal distribution function and

$$
\begin{aligned}
& d_{1}=\frac{x-\ln K+r_{f}(T-t)-\int_{t}^{T} \rho v_{u} \sigma_{u} d u+\frac{1}{2}\langle M\rangle_{T}}{\sqrt{\langle M\rangle_{T}}}, \\
& d_{2}=d_{1}-\sqrt{\langle M\rangle_{T}} .
\end{aligned}
$$

Moreover, if $\rho=0$, then since $\frac{1}{K} e^{x+r_{f}(T-t)} N^{\prime}\left(d_{1}\right)=N^{\prime}\left(d_{2}\right)$,

$$
\begin{aligned}
& \frac{\partial Q_{B S}}{\partial x}(t, x, \Gamma) \\
= & e^{x+\left(r_{f}-r_{d}\right)(T-t)} N\left(d_{1}\right) \\
& +e^{x+\left(r_{f}-r_{d}\right)(T-t)} N^{\prime}\left(d_{1}\right) \frac{\partial d_{1}}{\partial x}-K e^{\left(r_{f}-r_{d}\right)(T-t)} N^{\prime}\left(d_{2}\right) \frac{\partial d_{2}}{\partial x} \\
= & e^{x+\left(r_{f}-r_{d}\right)(T-t)} N\left(d_{1}\right) .
\end{aligned}
$$

We may write

$$
\begin{aligned}
c_{0}(t, x, v, \sigma) & =c(t, x, v, \sigma, 0,0,0) \\
& =E\left[e^{-r_{d}(T-t)} \max \left(e^{X_{T}}-K, 0\right)\right] \\
& =E\left[E\left[e^{-r_{d}(T-t)} \max \left(e^{X_{T}}-K, 0\right) \mid \mathcal{F}_{T}\right]\right] \\
& =E\left[e^{x+\left(r_{f}-r_{d}\right)(T-t)} N\left(d_{1}\right)-K e^{-r_{d}(T-t)} N\left(d_{2}\right)\right],
\end{aligned}
$$

where

$$
\begin{aligned}
& d_{1}=\frac{x-\ln K+r_{f}(T-t)+\frac{1}{2}\langle M\rangle_{T}}{\sqrt{\langle M\rangle_{T}}}, \\
& d_{2}=d_{1}-\sqrt{\langle M\rangle_{T}} .
\end{aligned}
$$

On the other hand, the equation (12) can be separated by following differential operators

$$
\begin{aligned}
L c= & \frac{1}{2}\left(v^{2} \frac{\partial^{2} c}{\partial x^{2}}+\xi_{1}^{2} v^{2} \frac{\partial^{2} c}{\partial v^{2}}+\xi_{2}^{2} \sigma^{2} \frac{\partial^{2} c}{\partial \sigma^{2}}\right)+\left(r_{f}-\frac{1}{2} v^{2}\right) \frac{\partial c}{\partial x} \\
& +\eta_{1} v \frac{\partial c}{\partial v}+\eta_{2} \sigma \frac{\partial c}{\partial \sigma}-r_{d} c
\end{aligned}
$$




$$
\begin{aligned}
\mathcal{A} c & =v \sigma \frac{\partial c}{\partial x}, & \mathcal{B} c & =\xi_{1} v^{2} \frac{\partial^{2} c}{\partial x \partial v}, \\
\mathcal{A}_{1} c & =\xi_{1} \xi_{2} v \sigma \frac{\partial^{2} c}{\partial v \partial \sigma}, & \mathcal{A}_{2} c & =\xi_{2} v \sigma \frac{\partial^{2} c}{\partial x \partial \sigma} .
\end{aligned}
$$

Then we can rewrite (12) in the following way

$$
\left\{\begin{array}{l}
\frac{\partial c}{\partial t}+L c-\rho \mathcal{A} c+\nu \mathcal{B} c+\nu \beta \rho \mathcal{A}_{1} c+\beta \rho \mathcal{A}_{2} c=0, \\
c(T, x, v, \sigma, \rho, \nu, \beta)=\max \left(e^{x}-K, 0\right) .
\end{array}\right.
$$

Consider the following first order Taylor series approximation

$$
\begin{aligned}
c(t, x, v, \sigma, \rho, \beta, \nu) \approx & c(t, x, v, \sigma, 0,0,0)+\rho \frac{\partial c}{\partial \rho}(t, x, v, \sigma, 0,0,0) \\
& +\nu \frac{\partial c}{\partial \nu}(t, x, v, \sigma, 0,0,0)+\beta \frac{\partial c}{\partial \beta}(t, x, v, \sigma, 0,0,0) .
\end{aligned}
$$

Put

$$
\begin{aligned}
c_{0}(t, x, v, \sigma) & =c(t, x, v, \sigma, 0,0,0), & c_{1}(t, x, v, \sigma) & =\frac{\partial c}{\partial \rho}(t, x, v, \sigma, 0,0,0), \\
\phi_{1}(t, x, v, \sigma) & =\frac{\partial c}{\partial \nu}(t, x, v, \sigma, 0,0,0), & \psi_{1}(t, x, v, \sigma) & =\frac{\partial c}{\partial \beta}(t, x, v, \sigma, 0,0,0),
\end{aligned}
$$

then we can rewrite approximation (18) as follows

$$
\begin{aligned}
c(t, x, v, \sigma, \rho, \beta, \nu) \approx & c_{0}(t, x, v, \sigma)+\rho c_{1}(t, x, v, \sigma) \\
& +\nu \phi_{1}(t, x, v, \sigma)+\beta \psi_{1}(t, x, v, \sigma) .
\end{aligned}
$$

By differentiating the equation (17) and for $(\rho, \nu, \beta)=(0,0,0)$, we can get the following PDE problems for $x \in \mathbf{R}, y, z>0$ and $t \in[0, T]$. Since the terminal condition of $\psi_{1}(t, x, v, \sigma)$ is zero, the $\mathrm{PDE}$ for $\psi_{1}(t, x, v, \sigma)$ does not exist. Actually, the correlation between the foreign exchange rate and its volatility $\beta$ does not affect to the stock price dynamics. Thus we exclude the series expansion for the correlation $\beta$. We just find the solutions for the following equations.

$$
\begin{aligned}
& \left\{\begin{aligned}
\frac{\partial c_{0}}{\partial t}+L c_{0}(t, x, v, \sigma) & =0, \\
c_{0}(T, x, v, \sigma) & =\max \left[e^{x}-K, 0\right],
\end{aligned}\right. \\
& \left\{\begin{aligned}
\frac{\partial c_{1}}{\partial t}+L c_{1}(t, x, v, \sigma) & =\mathcal{A} c_{0}(t, x, v, \sigma), \\
c_{1}(T, x, v, \sigma) & =0,
\end{aligned}\right. \\
& \left\{\begin{aligned}
\frac{\partial \phi_{1}}{\partial t}+L \phi_{1}(t, x, v, \sigma) & =-\mathcal{B} c_{0}(t, x, v, \sigma), \\
\phi_{1}(T, x, v, \sigma) & =0 .
\end{aligned}\right.
\end{aligned}
$$

We can find the solution $c_{0}$ directly from Black-Scholes option pricing formula. To solve inhomogeneous equations (20) and (21) we use Duhamel's 
principle which is a tool for finding solutions of inhomogeneous equation. Using Duhamel's principle we can represent the inhomogeneous equation as the integral of homogeneous equations.

Proposition 3.2. Let $c(t, x, v, \sigma, \rho, \beta, \nu)$ be a quanto option price at initial time $t$. Then the first order Taylor series expansion is

$$
c(t, x, v, \sigma, \rho, \beta, \nu) \approx c_{0}(t, x, v, \sigma)+\rho c_{1}(t, x, v, \sigma)+\nu \phi_{1}(t, x, v, \sigma)
$$

where

$$
\begin{aligned}
& c_{0}(t, x, v, \sigma)=E\left[e^{x+\left(r_{f}-r_{d}\right)(T-t)} N\left(d_{1}\right)-K e^{-r_{d}(T-t)} N\left(d_{2}\right)\right] \\
& c_{1}(t, x, v, \sigma)=-e^{-r_{d}(T-t)} \int_{t}^{T} E\left[v_{s} \sigma_{s} e^{X_{s}+r_{f}(T-s)} N\left(d_{1}\right)\right] d s \\
& \phi_{1}(t, x, v, \sigma)=-\frac{K \xi_{1} e^{-r_{d}(T-t)} d_{2} N^{\prime}\left(d_{2}\right)}{\langle M\rangle_{[t, T]}} \int_{t}^{T} E\left[v_{s}^{2} \int_{s}^{T} v_{u} \frac{\partial v_{u}}{\partial v} d u\right] d s .
\end{aligned}
$$

Proof. We can find directly $c_{0}(t, x, v, \sigma)$ from $c(t, x, v, \sigma, 0,0,0)$. We want to find the solution of the equation $(20)$,

$$
\left\{\begin{aligned}
\frac{\partial c_{1}}{\partial t}+L c_{1}(t, x, v, \sigma) & =\mathcal{A} c_{0}(t, x, v, \sigma) \\
c_{1}(T, x, v, \sigma) & =0
\end{aligned}\right.
$$

Since the above equation is the inhomogeneous PDE, by the Duhamel's principle (Lemma 2.1), we know that

$$
c_{1}(t, x, v, \sigma)=-\int_{t}^{T} p_{1}(t, x, v, \sigma ; s) d s,
$$

where $t \in[0, s), x \in \mathbf{R}, v, \sigma>0$ and $p_{1}(t, x, v, \sigma ; s)$ is a solution of

$$
\left\{\begin{aligned}
\frac{\partial p_{1}}{\partial t}(t, x, v, \sigma ; s)+L p_{1}(t, x, v, \sigma ; s) & =0 \\
p_{1}(s, x, v, \sigma ; s) & =\mathcal{A} c_{0}(s, x, v, \sigma) .
\end{aligned}\right.
$$

Using the Feynman Kaĉ formula, we can transform a PDE to an expectation of random variables. Applying the Feynman Kaĉ formula (Lemma 2.3) to the equation (23), we get the following expectation formula

$$
p_{1}(t, x, v, \sigma ; s)=E\left[e^{-r_{d}(s-t)} \mathcal{A} c_{0}\left(s, X_{s}, v_{s}, \sigma_{s}\right)\right] .
$$

The internal expectation is actually conditioned by general filtration up to time $s$. From equation (16),

$$
\begin{aligned}
\frac{\partial c_{0}}{\partial x}(t, x, v, \sigma) & =\frac{\partial}{\partial x} E\left[Q_{B S}\left(t, x+r_{f}(T-t), \sqrt{\langle M\rangle_{T}}\right)\right] \\
& =E\left[e^{x+\left(r_{f}-r_{d}\right)(T-t)} N\left(d_{1}\right)\right]
\end{aligned}
$$


we can compute $c_{1}$ as follows

$$
\begin{aligned}
& c_{1}(t, x, v, \sigma) \\
= & -\int_{t}^{T} E\left[e^{-r_{d}(s-t)} \mathcal{A} c_{0}\left(s, X_{s}, v_{s}, \sigma_{s}\right)\right] d s \\
= & -\int_{t}^{T} E\left[e^{-r_{d}(s-t)} v_{s} \sigma_{s} \frac{\partial c_{0}}{\partial x}\left(s, X_{s}, v_{s}, \sigma_{s}\right)\right] d s \\
= & -\int_{t}^{T} E\left[e^{-r_{d}(s-t)} v_{s} \sigma_{s} E\left[e^{X_{s}+\left(r_{f}-r_{d}\right)(T-s)} N\left(d_{1}\right)\right]\right] d s \\
= & -e^{-r_{d}(T-t)} \int_{t}^{T} E\left[v_{s} \sigma_{s} e^{X_{s}+r_{f}(T-s)} N\left(d_{1}\right)\right] d s .
\end{aligned}
$$

Similarly applying the Duhamel's principle to equation (21)

$$
\phi_{1}(t, x, v, s)=-\int_{t}^{T} q_{1}(t, x, v, \sigma ; s) d s,
$$

where $t \in[0, s), x \in \mathbf{R}, v, \sigma>0$ and $q_{1}(t, x, v, \sigma ; s)$ is a solution of

$$
\left\{\begin{aligned}
\frac{\partial q_{1}}{\partial t}(t, x, v, \sigma ; s)+L q_{1}(t, x, v, \sigma ; s) & =0, \\
q_{1}(s, x, v, \sigma ; s) & =-\mathcal{B} c_{0}(s, x, v, \sigma) .
\end{aligned}\right.
$$

Then by the Feynman-Kaĉ formula (Lemma 2.3)

$$
q_{1}(t, x, v, \sigma ; s)=E\left[e^{-r_{d}(s-t)}\left(-\mathcal{B} c_{0}\right)\left(s, X_{s}, v_{s}, \sigma_{s}\right)\right]
$$

and

$$
\begin{aligned}
\phi_{1}(t, x, v, s) & =-\int_{t}^{T} q_{1}(t, x, v, \sigma ; s) d s \\
& =\int_{t}^{T} E\left[e^{-r_{d}(s-t)} \mathcal{B} c_{0}\left(s, X_{s}, v_{s}, \sigma_{s}\right)\right] d s \\
& =\int_{t}^{T} E\left[e^{-r_{d}(s-t)} \xi_{1} v_{s}^{2} \frac{\partial^{2} c_{0}}{\partial x \partial v}\left(s, X_{s}, v_{s}, \sigma_{s}\right)\right] d s,
\end{aligned}
$$

we can calculate the partial derivative of $c_{0}(t, x, v, \sigma)$ for $v$ as follows

$$
\begin{aligned}
& \frac{\partial}{\partial v} c_{0}(t, x, v, \sigma) \\
= & e^{x+\left(r_{f}-r_{d}\right)(T-t)} N^{\prime}\left(d_{1}\right) \frac{\partial d_{1}}{\partial v}-K e^{-r_{d}(T-t)} N^{\prime}\left(d_{2}\right) \frac{\partial d_{2}}{\partial v} \\
= & N^{\prime}\left(d_{2}\right) K e^{-r_{d}(T-t)}\left(\frac{\partial}{\partial v} d_{1}-\frac{\partial}{\partial v} d_{2}\right) \\
= & K e^{-r_{d}(T-t)} N^{\prime}\left(d_{2}\right) \frac{\partial}{\partial v} \sqrt{\langle M\rangle_{T}} .
\end{aligned}
$$


Here

$$
\begin{aligned}
\frac{\partial}{\partial v} \sqrt{\langle M\rangle_{T}} & =\frac{1}{2}\langle M\rangle_{T}^{-\frac{1}{2}} \frac{\partial}{\partial v}\langle M\rangle_{T} \\
& =\frac{1}{2 \sqrt{\langle M\rangle_{T}}} \frac{\partial}{\partial v} \int_{t}^{T} v_{u}^{2} d u \\
& =\frac{1}{\sqrt{\langle M\rangle_{T}}} \int_{t}^{T} v_{u} \frac{\partial v_{u}}{\partial v} d u .
\end{aligned}
$$

Thus

$$
\begin{aligned}
\frac{\partial^{2}}{\partial x \partial v} Q_{B S}\left(t, x, \sqrt{\langle M\rangle_{T}}\right) & =\frac{\partial}{\partial x}\left(K e^{-r_{d}(T-t)} N^{\prime}\left(d_{2}\right) \frac{\partial}{\partial v} \sqrt{\langle M\rangle_{T}}\right) \\
& =N^{\prime \prime}\left(d_{2}\right) \frac{\partial d_{2}}{\partial x} K e^{-r_{d}(T-t)} \frac{\partial}{\partial v} \sqrt{\langle M\rangle_{T}} \\
& =\frac{-d_{2} N^{\prime}\left(d_{2}\right)}{\sqrt{\langle M\rangle_{T}}} K e^{-r_{d}(T-t)} \frac{\partial}{\partial v} \sqrt{\langle M\rangle_{T}} \\
& =K e^{-r_{d}(T-t)} \frac{-d_{2} N^{\prime}\left(d_{2}\right)}{\langle M\rangle_{T}} \int_{t}^{T} v_{u} \frac{\partial v_{u}}{\partial v} d u .
\end{aligned}
$$

Therefore,

$$
\begin{aligned}
& \phi_{1}(t, x, v, \sigma) \\
= & \int_{t}^{T} e^{-r_{d}(s-t)} E\left[\xi_{1} v_{s}^{2} E\left[\frac{\partial^{2}}{\partial x \partial v} Q_{B S}\left(s, X_{s}, \sqrt{\langle M\rangle_{[s, T]}}\right)\right]\right] \\
= & \int_{t}^{T} e^{-r_{d}(s-t)} E\left[\xi_{1} v_{s}^{2} E\left[K e^{-r_{d}(T-s)} \frac{-d_{2} N^{\prime}\left(d_{2}\right)}{\langle M\rangle_{[s, T]}} \int_{s}^{T} v_{u} \frac{\partial v_{u}}{\partial v} d u\right]\right] d s \\
= & \int_{t}^{T} K e^{-r_{d}(T-t)} E\left[\xi_{1} v_{s}^{2} E\left[\frac{-d_{2} N^{\prime}\left(d_{2}\right)}{\langle M\rangle_{[s, T]}} \int_{s}^{T} v_{u} \frac{\partial v_{u}}{\partial v} d u\right]\right] d s .
\end{aligned}
$$

Let $\mathcal{F}_{T}$ be a filtration generated by $v_{u}$ and $\sigma_{u}(t \leq u \leq T)$. Then

$$
\begin{aligned}
& \int_{t}^{T} K e^{-r_{d}(T-t)} E\left[\xi_{1} v_{s}^{2} E\left[\frac{-d_{2} N^{\prime}\left(d_{2}\right)}{\langle M\rangle_{[s, T]}} \int_{s}^{T} v_{u} \frac{\partial v_{u}}{\partial v} d u\right]\right] d s \\
= & -\int_{t}^{T} K e^{-r_{d}(T-t)} E\left[\xi_{1} v_{s}^{2} \frac{d_{2} N^{\prime}\left(d_{2}\right)}{\langle M\rangle_{[s, T]}} \int_{s}^{T} v_{u} \frac{\partial v_{u}}{\partial v} d u\right] d s \\
= & -\int_{t}^{T} K e^{-r_{d}(T-t)} E\left[\frac{\xi_{1} v_{s}^{2}}{\langle M\rangle_{[s, T]}} \int_{s}^{T} v_{u} \frac{\partial v_{u}}{\partial v} d u E\left[d_{2} N^{\prime}\left(d_{2}\right) \mid \mathcal{F}_{T}\right]\right] d s,
\end{aligned}
$$

where

$$
d_{2}=d_{2}\left(s, X_{s}, \sqrt{\langle M\rangle_{[s, T]}}\right)=\frac{X_{s}-\ln K+r_{f}(T-s)-\frac{1}{2}\langle M\rangle_{[s, T]}}{\sqrt{\langle M\rangle_{[s, T]}}} .
$$


We already know that

$$
X_{s} \mid \mathcal{F}_{T} \sim N\left(x-\ln K+r_{f}(s-t)-\frac{1}{2}\langle M\rangle_{[t, s]},\langle M\rangle_{[t, s]}\right) .
$$

Hence $d_{2}$ is distributed as follow

$$
d_{2} \mid \mathcal{F}_{T} \sim N\left(\frac{x-\ln K+r_{f}(T-t)-\frac{1}{2}\langle M\rangle_{[t, T]}}{\langle M\rangle_{[s, T]}}, \frac{\langle M\rangle_{[t, s]}}{\langle M\rangle_{[s, T]}}\right) .
$$

If $z \sim N(\mu, \sigma)$, then

$$
E\left[z N^{\prime}(z)\right]=\frac{\mu}{\sqrt{2 \pi}\left(1+\sigma^{2}\right)^{3 / 2}} e^{-\frac{\mu^{2}}{2\left(1+\sigma^{2}\right)}} .
$$

Since

$$
E\left[d_{2}\left(s, X_{s}, \sqrt{\langle M\rangle_{[s, T]}}\right) \mid \mathcal{F}_{T}\right]=d_{2}(t, x, v, \sigma) \times \frac{\langle M\rangle_{[s, T]}}{\langle M\rangle_{[t, T]}}
$$

by using the formula (25)

$$
\begin{aligned}
& E\left[d_{2}\left(s, X_{s}, \sqrt{\langle M\rangle_{[s, T]}}\right) N^{\prime}\left(d_{2}\left(s, X_{s}, \sqrt{\langle M\rangle_{[s, T]}}\right)\right) \mid \mathcal{F}_{T}\right] \\
= & \frac{\langle M\rangle_{[s, T]}}{\langle M\rangle_{[t, T]}} d_{2}\left(t, x, \sqrt{\langle M\rangle_{[t, T]}}\right) N^{\prime}\left(d_{2}\left(t, x, \sqrt{\langle M\rangle_{[t, T]}}\right)\right)
\end{aligned}
$$

we can calculate $\phi_{1}$ as follow

$$
\begin{aligned}
& \phi_{1}(t, x, v, \sigma) \\
= & -\int_{t}^{T} \frac{K e^{-r_{d}(T-t)} d_{2} N^{\prime}\left(d_{2}\right)}{\langle M\rangle_{[t, T]}} E\left[\xi_{1} v_{s}^{2} \int_{s}^{T} v_{u} \frac{\partial v_{u}}{\partial v} d u\right] d s \\
= & -\frac{K \xi_{1} e^{-r_{d}(T-t)} d_{2} N^{\prime}\left(d_{2}\right)}{\langle M\rangle_{[t, T]}} \int_{t}^{T} E\left[v_{s}^{2} \int_{s}^{T} v_{u} \frac{\partial v_{u}}{\partial v} d u\right] d s .
\end{aligned}
$$

Since $\langle M\rangle_{T}$ is a random variable sufficiently concentrated around its mean in general, one may think of approximating it by its expectation. This idea was first introduced by Alós [1]. The next theorem is the main result of the paper.

Theorem 3.3. Replacing $\langle M\rangle_{T}$ by $E\left[\langle M\rangle_{T}\right]$ in Proposition 3.2, the approximate quanto option formula becomes

$$
c(t, x, v, \sigma, \rho, \beta, \nu) \approx \overline{c_{0}}(t, x, v, \sigma)+\rho \overline{c_{1}}(t, x, v, \sigma)+\nu \overline{\phi_{1}}(t, x, v, \sigma),
$$

where

$$
\begin{aligned}
& \overline{c_{0}}(t, x, v, \sigma)=e^{x+\left(r_{f}-r_{d}\right)(T-t)} N\left(\bar{d}_{1}\right)-K e^{-r_{d}(T-t)} N\left(\bar{d}_{2}\right), \\
& \overline{c_{1}}(t, x, v, \sigma)=-\frac{e^{x+\left(r_{f}-r_{d}\right)(T-t)} N\left(\bar{d}_{1}\right) v \sigma}{\eta_{1}+\eta_{2}+\frac{1}{2}\left(\xi_{1}^{2}+\xi_{2}^{2}\right)}\left(e^{\left(\eta_{1}+\eta_{2}+\frac{1}{2}\left(\xi_{1}^{2}+\xi_{2}^{2}\right)\right)(T-t)}-1\right),
\end{aligned}
$$




$$
\bar{\phi}_{1}(t, x, v, \sigma)=-\frac{K e^{-r_{d}(T-t)} \bar{d}_{2} N^{\prime}\left(\bar{d}_{2}\right) \xi_{1} v}{2 \eta_{1}+\xi_{1}^{2}}\left(e^{\left(2 \eta_{1}+\xi_{1}^{2}\right)(T-t)}-1\right) .
$$

Proof. If we replace $\langle M\rangle_{T}$ by $E\left[\langle M\rangle_{T}\right]$, then we rewrite $d_{1}$ by $\bar{d}_{1}$. We have to calculate $E\left[e^{X_{s}+r_{f}(T-s)} N\left(d_{1}\left(s, X_{s}, \sqrt{\langle M\rangle_{[s, T]}}\right)\right)\right]$ in equation $c_{1}(t, x, v, \sigma)$, but we take an expectation of random variable $X_{s}$ instead of solving the expectation as follow

$$
E\left[e^{X_{s}+r_{f}(T-s)}\right]=e^{x+r_{f}(T-t)} .
$$

Otherwise we cannot figure out the expectation problem. Thus we rewrite $c_{1}$ and $\phi_{1}$ like follows

$$
\overline{c_{1}}(t, x, v, \sigma)=-e^{x+\left(r_{f}-r_{d}\right)(T-t)} \int_{t}^{T} E\left[v_{s} \sigma_{s}\right] N\left(\bar{d}_{1}(s)\right) d s,
$$

$$
\bar{\phi}_{1}(t, x, v, \sigma)=-\frac{K \xi_{1} e^{-r_{d}(T-t)} \bar{d}_{2}(t) N^{\prime}\left(\bar{d}_{2}(t)\right)}{\langle\bar{M}\rangle_{[t, T]}} \int_{t}^{T} E\left[v_{s}^{2} \int_{s}^{T} v_{u} \frac{\partial v_{u}}{\partial v} d u\right] d s,
$$

where, for $t \leq u \leq T$

$$
\begin{aligned}
\bar{d}_{i}(u) & =d_{i}\left(u, X_{u}, \sqrt{\langle\bar{M}\rangle_{[u, T]}}\right) \quad i=1,2, \\
\langle\bar{M}\rangle_{[u, T]} & =E\left[\langle M\rangle_{[u, T]}\right] .
\end{aligned}
$$

In the equation (26), we have to calculate the integral of $N\left(\bar{d}_{1}\right)$ but $N(\cdot)$ is an integral form itself. It is difficult to handle this formula, so we can choose an adjustment factor $\alpha$ which is approximate the integral of $N\left(\bar{d}_{1}\right)$ such that

$$
\int_{t}^{T} E\left[v_{s} \sigma_{s}\right] N\left(\bar{d}_{1}(s)\right) d s=N\left(\bar{d}_{1}(\alpha)\right) \int_{t}^{T} E\left[v_{s} \sigma_{s}\right] d s .
$$

Therefore, for some $\alpha \geq 0$

$$
\overline{c_{1}}(t, x, v, \sigma)=-e^{x+\left(r_{f}-r_{d}\right)(T-t)} N\left(\bar{d}_{1}(\alpha)\right) \int_{t}^{T} E\left[v_{s} \sigma_{s}\right] d s .
$$

In the risk-netral world and the uncorrelated case $(\nu=0$ and $\beta=0)$ with $X_{t}=\ln S_{t}$, we have

$$
\begin{aligned}
d X_{t} & =\left(r_{f}-\rho v_{t} \sigma_{t}-\frac{1}{2} v_{t}^{2}\right) d t+v_{t} d W_{t}, \\
d v_{t} & =\eta_{1} v_{t} d t+\xi_{1} v_{t} d B_{t} \\
d \sigma_{t} & =\eta_{2} \sigma_{t} d t+\xi_{2} \sigma_{t} d \tilde{B}_{t} .
\end{aligned}
$$

Hence, the volatilities are

$$
\begin{aligned}
v_{u} & =v e^{\left(\eta_{1}-\frac{1}{2} \xi_{1}^{2}\right)(u-t)+\xi_{1}\left(B_{u}-B_{t}\right)}, \\
\sigma_{u} & =\sigma e^{\left(\eta_{2}-\frac{1}{2} \xi_{2}^{2}\right)(u-t)+\xi_{2}\left(\tilde{B_{u}}-\tilde{B}_{t}\right)}
\end{aligned}
$$


and

$$
\begin{aligned}
E\left[v_{u}^{2}\right] & =E\left[v^{2} e^{2\left(\eta_{1}-\frac{1}{2} \xi_{1}^{2}\right)(u-t)+2 \xi_{1}\left(B_{u}-B_{t}\right)}\right] \\
& =v^{2} e^{2\left(\eta_{1}-\frac{1}{2} \xi_{1}^{2}\right)(u-t)} E\left[e^{2 \xi_{1}\left(B_{u}-B_{t}\right)}\right] \\
& =v^{2} e^{2\left(\eta_{1}-\frac{1}{2} \xi_{1}^{2}\right)(u-t)} e^{2 \xi_{1}^{2}(u-t)} \\
& =v^{2} e^{\left(2 \eta_{1}+\xi_{1}^{2}\right)(u-t)} .
\end{aligned}
$$

This leads to the following approximations

$$
\begin{aligned}
E\left[\langle M\rangle_{[s, T]}\right] & =E\left[\int_{s}^{T} v_{u}^{2} d u\right] \\
& =\int_{s}^{T} E\left[v_{u}^{2}\right] d u \\
& =\int_{s}^{T} v^{2} e^{\left(2 \eta_{1}+\xi_{1}^{2}\right)(u-t)} d u \\
& =v^{2} \frac{e^{\left(2 \eta_{1}+\xi_{1}^{2}\right)(T-t)}-e^{\left(2 \eta_{1}+\xi_{1}^{2}\right)(s-t)}}{2 \eta_{1}+\xi_{1}^{2}} .
\end{aligned}
$$

If we apply the above equation to (26) and (27) then we can find the followings

$$
\begin{aligned}
& \overline{c_{0}}(t, x, v, \sigma)=e^{x+\left(r_{f}-r_{d}\right)(T-t)} N\left(\bar{d}_{1}\right)-K e^{-r_{d}(T-t)} N\left(\bar{d}_{2}\right), \\
& \overline{c_{1}}(t, x, v, \sigma)=-\frac{e^{x+\left(r_{f}-r_{d}\right)(T-t)} N\left(\bar{d}_{1}\right) v \sigma}{\eta_{1}+\eta_{2}+\frac{1}{2}\left(\xi_{1}^{2}+\xi_{2}^{2}\right)}\left(e^{\left(\eta_{1}+\eta_{2}+\frac{1}{2}\left(\xi_{1}^{2}+\xi_{2}^{2}\right)\right)(T-t)}-1\right), \\
& \bar{\phi}_{1}(t, x, v, \sigma)=-\frac{K e^{-r_{d}(T-t)} \bar{d}_{2} N^{\prime}\left(\bar{d}_{2}\right) \xi_{1} v}{2 \eta_{1}+\xi_{1}^{2}}\left(e^{\left(2 \eta_{1}+\xi_{1}^{2}\right)(T-t)}-1\right),
\end{aligned}
$$

where

$$
\begin{aligned}
E\left[v_{s} \sigma_{s}\right] & =E\left[v \sigma e^{\left(\eta_{1}+\eta_{2}-\frac{1}{2}\left(\xi_{1}^{2}+\xi_{2}^{2}\right)\right)(s-t)+\xi_{1}\left(B_{s}-B_{t}\right)+\xi_{2}\left(\tilde{B_{s}}-\tilde{B}_{t}\right)}\right] \\
& =v \sigma e^{\left(\eta_{1}+\eta_{2}-\frac{1}{2}\left(\xi_{1}^{2}+\xi_{2}^{2}\right)\right)(s-t)} e^{\left(\xi_{1}^{2}+\xi_{2}^{2}\right)(s-t)} \\
& =v \sigma e^{\left(\eta_{1}+\eta_{2}+\frac{1}{2}\left(\xi_{1}^{2}+\xi_{2}^{2}\right)\right)(s-t)} \\
\int_{t}^{T} E\left[v_{s} \sigma_{s}\right] d s & =\int_{t}^{T} v \sigma e^{\left(\eta_{1}+\eta_{2}+\frac{1}{2}\left(\xi_{1}^{2}+\xi_{2}^{2}\right)\right)(s-t)} d s \\
& =v \sigma \frac{e^{\left(\eta_{1}+\eta_{2}+\frac{1}{2}\left(\xi_{1}^{2}+\xi_{2}^{2}\right)\right)(T-t)}-1}{\eta_{1}+\eta_{2}+\frac{1}{2}\left(\xi_{1}^{2}+\xi_{2}^{2}\right)}
\end{aligned}
$$

and

$$
\frac{\partial v_{u}}{\partial v}=e^{\left(\eta_{1}-\frac{1}{2} \xi_{1}^{2}\right)(u-t)+\xi_{1}\left(B_{u}-B_{t}\right)}
$$




$$
\begin{aligned}
E\left[\int_{s}^{T} v_{u} \frac{\partial v_{u}}{\partial v} d u\right] & =E\left[\int_{s}^{T} v e^{2\left(\eta_{1}-\frac{1}{2} \xi_{1}^{2}\right)(u-t)+2 \xi_{1}\left(B_{u}-B_{t}\right)} d u\right] \\
& =\int_{s}^{T} v e^{2\left(\eta_{1}-\frac{1}{2} \xi_{1}^{2}\right)(u-t)} E\left[e^{2 \xi_{1}\left(B_{u}-B_{t}\right)}\right] d u \\
& =\int_{s}^{T} v e^{\left(2 \eta_{1}+\xi_{1}^{2}\right)(u-t)} d u \\
& =v \frac{e^{\left(2 \eta_{1}+\xi_{1}^{2}\right)(T-t)}-e^{\left(2 \eta_{1}+\xi_{1}^{2}\right)(s-t)}}{2 \eta_{1}+\xi_{1}^{2}}
\end{aligned}
$$

\section{Numerical examples}

Now we can find the approximated value of the quanto option price by using the formula in Theorem 3.3. Using the main theorem, we choose the half value of the time to maturity as the adjustment factor $\alpha$. We compare the result between the approximation value and the Monte Carlo simulation value. We suppose that there is a quanto European call option of S\&P500 index with strike 1,200 and maturity date 13-Jun-2011. The information of the quanto option is showed in Table 1.

TABLE 1. Quanto option information sample

\begin{tabular}{c|c}
\hline \multicolumn{2}{c}{ Information } \\
\hline Name & Quanto option sample \\
\hline Underlying & S\&P500 \\
\hline Issue Volume & 100 \\
\hline Amount per point in [Currency] & $50[\mathrm{USD}]$ \\
\hline Currency / quote & KRW / Unit \\
\hline Option Type & European Call \\
\hline Strike Value & 1,200 \\
\hline Exchange Rate & $1,100($ KRW /USD) \\
\hline Maturity Date & $2011-06-13$ \\
\hline
\end{tabular}

The model parameters are set to Table 2. The data are viewed at 2010-1013. The number of the sample data which are used in making the constant volatilities and correlations is 250 from the view date. The volatilities and correlations are calculated by the moving average method which is the same as computing the standard deviation of historical market data. We use the USD riskless rate as 1 year USD LIBOR and the KRW riskless rate as 1 year KRW treasury bond rate at the view date. 
TABLE 2. Market Data Set

\begin{tabular}{c|c}
\hline \multicolumn{2}{c}{ Data set } \\
\hline View Date & $2010-10-13$ \\
\hline S\&P500 & $1,169.77$ \\
\hline FX Rate (KRW/USD) & 1,127 \\
\hline Volatility of S\&P500 & $18.58 \%$ \\
\hline Volatility of FX Rate & $11.83 \%$ \\
\hline Correlation between S\&P500 and its volatility & -0.55 \\
\hline Volatility of volatility of S\&P500 & $11.72 \%$ \\
\hline Volatility of volatility of FX Rate & $16.8 \%$ \\
\hline USD LIBOR(1Y) & $0.77 \%$ \\
\hline KRW Treasury Rate(1Y) & $2.91 \%$ \\
\hline
\end{tabular}

The Monte Carlo estimates of the price were obtained by simulating 250,000 paths with 1,000 time-grid points on the interval $[0, T]$. From the Table 3 to the Table 7 , the upper value is from our approximation formula, the lower value is from Monte Carlo simulation method and the right side of each entry percent point is a ratio between the approximation value and the simulation value. In the Table 3 that is the first example, we fix all the correlations zero $(\rho=\nu=0)$ and move the time to maturity and the strike value. In the Table 4 , time to maturity is 1 and correlation $\nu$ is 0 . We move the correlation $\rho$ and the strike price. In the Table 5,6 , and 7 , we fix the correlation between stock and stock volatility $\nu=-0.55$ and move the correlation $\rho$ and the strike price $K$. The prices of our approximation method and the Monte Carlo method are calculated in domestic currency. In our approximation method, we first calculate the option price $c(t, x, v, \sigma, \rho, \beta, \nu)$ in foreign currency then we multiply the predetermined exchange rate to the price.

TABLE 3. Monte Carlo estimations with zero correlations

\begin{tabular}{|c|c|c|c|c|c|c|}
\hline$T \backslash K$ & \multicolumn{2}{|c|}{1100} & \multicolumn{2}{|c|}{1150} & \multicolumn{2}{|c|}{1200} \\
\hline 0.25 & $\begin{array}{l}478,095,931 \\
478,511,402\end{array}$ & $(0.1 \%)$ & $\begin{array}{l}300,060,155 \\
301,560,208\end{array}$ & $(0.5 \%)$ & $\begin{array}{l}171,427,534 \\
172,055,249\end{array}$ & $(0.4 \%)$ \\
\hline 0.5 & $\begin{array}{l}563,712,483 \\
562,721,490\end{array}$ & $(0.2 \%)$ & $\begin{array}{l}400,465,433 \\
397,836,079\end{array}$ & $(0.7 \%)$ & $\begin{array}{l}272,467,837 \\
272,569,146\end{array}$ & $(0.0 \%)$ \\
\hline 1 & $\begin{array}{l}693,286,693 \\
691,414,566\end{array}$ & $(0.3 \%)$ & $\begin{array}{l}543,019,112 \\
540,906,951\end{array}$ & $(0.4 \%)$ & $\begin{array}{l}417,477,621 \\
413,576,358\end{array}$ & $(1 \%)$ \\
\hline
\end{tabular}


TABLE $4 . T=1$ and correlation $\nu=0$

\begin{tabular}{c|cc|cc|cc}
\hline$\rho \backslash K$ & \multicolumn{3}{|c|}{1100} & \multicolumn{2}{|c|}{1150} & \multicolumn{2}{c}{1200} \\
\hline \multirow{2}{*}{0.4} & $733,707,680$ & $(0.3 \%)$ & $576,592,257$ & $(0.3 \%)$ & $443,918,065$ & \\
& $731,661,695$ & & $574,916,009$ & & $446,471,872$ & $(0.6 \%)$ \\
\hline \multirow{2}{*}{0.2} & $713,497,186$ & $(0.6 \%)$ & $559,805,685$ & $(0.2 \%)$ & $430,697,843$ & $(0.8 \%)$ \\
& $709,296,252$ & & $560,643,025$ & & $427,309,852$ & \\
\hline \multirow{2}{*}{0} & $693,286,692$ & $(0.5 \%)$ & $543,019,112$ & $(0.0 \%)$ & $417,477,621$ & $(0.3 \%)$ \\
& $696,555,194$ & & $542,959,975$ & & $418,666,951$ & \\
\hline \multirow{2}{*}{0.2} & $673,076,198$ & $(0.3 \%)$ & $526,232,539$ & $(0.3 \%)$ & $404,257,398$ & $(0.3 \%)$ \\
& $671,322,203$ & & $527,710,228$ & & $402,994,168$ & \\
\hline \multirow{2}{*}{0.4} & $652,865,704$ & $(0.2 \%)$ & $509,445,966$ & $(0.1 \%)$ & $391,037,176$ & $(0.7 \%)$ \\
& $654,211,596$ & & $508,860,038$ & & $388,483,249$ & \\
\hline
\end{tabular}

TABLE 5. $T=1$ and correlation $\nu=-0.55$

\begin{tabular}{|c|c|c|c|c|c|c|}
\hline$\rho \backslash K$ & \multicolumn{2}{|c|}{1100} & \multicolumn{2}{|c|}{1150} & \multicolumn{2}{|c|}{1200} \\
\hline-0.4 & $\begin{array}{l}741,267,477 \\
735,422,927\end{array}$ & $(0.8 \%)$ & $\begin{array}{l}577,758,979 \\
576,855,830\end{array}$ & $(0.2 \%)$ & $\begin{array}{l}438,198,881 \\
441,735,145\end{array}$ & $(0.8 \%)$ \\
\hline-0.2 & $\begin{array}{l}721,056,983 \\
711,396,749\end{array}$ & $(1.3 \%)$ & $\begin{array}{l}560,972,406 \\
557,444,046\end{array}$ & $(0.6 \%)$ & $\begin{array}{l}424,978,659 \\
426,850,549\end{array}$ & $(0.4 \%)$ \\
\hline 0 & $\begin{array}{l}700,846,489 \\
693,801,357\end{array}$ & $(1.0 \%)$ & $\begin{array}{l}544,185,833 \\
540,942,983\end{array}$ & $(0.6 \%)$ & $\begin{array}{l}411,758,436 \\
412,306,861\end{array}$ & $(0.1 \%)$ \\
\hline 0.2 & $\begin{array}{l}680,635,995 \\
678,278,286\end{array}$ & $(0.4 \%)$ & $\begin{array}{l}527,399,260 \\
523,628,976\end{array}$ & $(0.7 \%)$ & $\begin{array}{l}398,538,214 \\
401,668,459\end{array}$ & $(0.8 \%)$ \\
\hline 0.4 & $\begin{array}{l}660,425,501 \\
662,302,097\end{array}$ & $(0.3 \%)$ & $\begin{array}{l}510,612,687 \\
509,932,660\end{array}$ & $(0.1 \%)$ & $\begin{array}{l}385,317,992 \\
385,985,981\end{array}$ & $(0.2 \%)$ \\
\hline
\end{tabular}

TABLE $6 . T=0.5$ and correlation $\nu=-0.55$

\begin{tabular}{|c|c|c|c|c|c|c|}
\hline$\rho \backslash K$ & \multicolumn{2}{|c|}{1100} & \multicolumn{2}{|c|}{1150} & \multicolumn{2}{|c|}{1200} \\
\hline-0.4 & $\begin{array}{l}591,062,599 \\
585,208,735\end{array}$ & $(1.0 \%)$ & $\begin{array}{l}418,916,637 \\
418,990,946\end{array}$ & $(0.0 \%)$ & $\begin{array}{l}280,957,939 \\
282,192,769\end{array}$ & $(0.4 \%)$ \\
\hline-0.2 & $\begin{array}{l}580,193,189 \\
575,986,769\end{array}$ & $(0.7 \%)$ & $\begin{array}{l}410,382,665 \\
409,915,669\end{array}$ & $(0.1 \%)$ & $\begin{array}{l}274,963,478 \\
276,180,713\end{array}$ & $(0.4 \%)$ \\
\hline 0 & $\begin{array}{l}569,323,779 \\
564,492,060\end{array}$ & $(0.9 \%)$ & $\begin{array}{l}401,848,692 \\
399,865,410\end{array}$ & $(0.5 \%)$ & $\begin{array}{l}268,969,017 \\
269,768,467\end{array}$ & $(0.3 \%)$ \\
\hline 0.2 & $\begin{array}{l}558,454,369 \\
557,417,509\end{array}$ & $(0.2 \%)$ & $\begin{array}{l}393,314,720 \\
392,798,704\end{array}$ & $(0.1 \%)$ & $\begin{array}{l}262,974,557 \\
264,546,984\end{array}$ & $(0.6 \%)$ \\
\hline 0.4 & $\begin{array}{l}547,584,959 \\
545,888,148\end{array}$ & $(0.3 \%)$ & $\begin{array}{l}384,780,747 \\
385,181,826\end{array}$ & $(0.1 \%)$ & $\begin{array}{l}256,980,096 \\
258,396,671\end{array}$ & $(0.6 \%)$ \\
\hline
\end{tabular}


TABLE 7. $T=0.25$ and correlation $\nu=-0.55$

\begin{tabular}{|c|c|c|c|c|c|c|}
\hline$\rho \backslash K$ & \multicolumn{2}{|c|}{1100} & \multicolumn{2}{|c|}{1150} & \multicolumn{2}{|c|}{1200} \\
\hline-0.4 & $\begin{array}{l}493,683,947 \\
491,028,807\end{array}$ & $(0.5 \%)$ & $\begin{array}{l}310,057,723 \\
309,051,984\end{array}$ & $(0.3 \%)$ & $\begin{array}{l}174,428,313 \\
175,726,849\end{array}$ & $(0.7 \%)$ \\
\hline-0.2 & $\begin{array}{l}487,756,160 \\
484,732,343\end{array}$ & $(0.6 \%)$ & $\begin{array}{l}305,643,449 \\
303,809,470\end{array}$ & $(0.6 \%)$ & $\begin{array}{l}171,803,006 \\
173,237,309\end{array}$ & $(0.8 \%)$ \\
\hline 0 & $\begin{array}{l}481,828,372 \\
479,168,812\end{array}$ & $(0.6 \%)$ & $\begin{array}{l}301,229,174 \\
300,851,576\end{array}$ & $(0.1 \%)$ & $\begin{array}{l}169,177,699 \\
168,923,701\end{array}$ & $(0.2 \%)$ \\
\hline 0.2 & $\begin{array}{l}475,900,585 \\
473,986,942\end{array}$ & $(0.4 \%)$ & $\begin{array}{l}296,814,899 \\
295,859,291\end{array}$ & $(0.3 \%)$ & $\begin{array}{l}166,552,393 \\
167,293,256\end{array}$ & $(0.4 \%)$ \\
\hline 0.4 & $\begin{array}{l}469,972,798 \\
471,260,262\end{array}$ & $(0.3 \%)$ & $\begin{array}{l}292,400,624 \\
292,879,602\end{array}$ & $(0.2 \%)$ & $\begin{array}{l}163,927,086 \\
163,996,976\end{array}$ & $(0.0 \%)$ \\
\hline
\end{tabular}

From the result tables, the difference ratio between our series expansion formula result and the Monte Carlo simulation result is less then $1.3 \%$. This results show that our series expansion formula value is very close to the expected present value of the quanto option payoff. Monte Carlo simulation method is common used in pricing or calculating financial product which is not known its closed form formula. But this method is too much computation time needed for obtaining accurate pricing value. In calculating the quanto option value with the Hull and White stochastic volatility model, the correlation expansion method is a nice alternative to the Monte Carlo simulation method.

\section{References}

[1] E. Alós, A generalization of the Hull and White formula with application to option pricing approximation, Finance Stoch. 10 (2006), no. 3, 353-365.

[2] F. Antonelli and S. Scarlatti, Pricing option under stochastic volatility. A power series approach, Finance Stoch. 13 (2009), no. 2, 269-303.

[3] F. Antonelli, A. Ranponi, and S. Scarlatti, Exchange option pricing under stochastic volatility: a correlation expansion, Review of Derivatives Research 13 (2010), 45-73.

[4] C. C. Ball and A. Roma, Stochastic volatility option pricing, The Journal of Financial and Quantitative Analysis 29 (1994), 581-607.

[5] S. Heston, A closed-form solution for options with stochastic volatility with applications to bond and currency options, The Review of Financial Studies 6 (1993), 327-343.

[6] J. C. Hull and A. White, The pricing of options on assets with stochastic volatilities, J. Finance 2 (1987), 281-300.

[7] E. Stein and J. Stein, Stock price distributions with stochastic volatility: an analytic approach, The Review of Financial Studies 4 (1991), 727-752.

JiHO PARK

Department of Mathematics

SOGANG University

SeOul 121-742, Korea

E-mail address: hetalk@sogang.ac.kr 
YOUNGROK LEE

Department of Mathematics

Sogang University

SEOUl 121-742, Korea

E-mail address: roke4208@sogang.ac.kr

JAESUng LeE

Department of Mathematics

SOGANG University

SeOul 121-742, Korea

E-mail address: jalee@sogang.ac.kr 